\title{
Diskurse öffnen, Grenzen überwinden: Der Völkerrechtsblog als Plattform für globale Wissenschaftskommunikation im digitalen Zeitalter
}

\author{
Raffaela Kunz/Michael Riegner/Dana Schmalz"
}

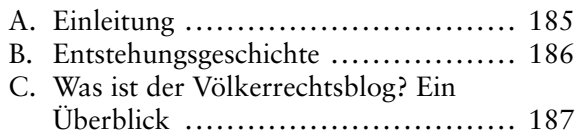

D. Zukunftsvisionen ...................... 190

\section{A. Einleitung}

Obwohl Digitalisierung und Neue Medien längst auch die traditionsreiche Rechtswissenschaft erreicht haben, herrscht auch hier im Gegensatz zu den Naturwissenschaften bekanntlich nach wie vor eine gewisse „geisteswissenschaftliche Netzresistenz". ${ }^{1}$ Zwar sind positive Effekte für die internationale Sichtbarkeit, Rezeption und Zitierhäufigkeit von elektronischen und frei zugänglichen Publikationsformen längst auch für die Rechtswissenschaft nachgewiesen; ${ }^{2}$ auch der Wissenschaftsrat hat darauf aufmerksam gemacht, dass sich die Geistes- und Sozialwissenschaften an Intrastrukturentwicklungen anpassen müssten, um international anschlussfähig zu bleiben. ${ }^{3}$ In seinem Bericht zur Zukunft der Rechtswissenschaft weist der Rat zudem ausdrücklich auf die Wichtigkeit elektronischen Publizierens für die internationale Sichtbarkeit hin. ${ }^{4}$ Trotzdem hat Open Access in der Rechtswissenschaft im deutschsprachigen Raum nach wie vor einen schweren Stand und stößt zuweilen gar auf Widerstand: Als die Universität Konstanz versuchte, ihre Wissenschaftlerinnen und Wissenschaftler zur Wahrnehmung ihres Rechts auf Zweitveröffentlichung zu verpflichten und die Texte frei zugänglich zu machen, waren es bekanntlich Mitglieder der Juristischen Fakultät, die unter Berufung auf ihre Wissenschaftsfreiheit gerichtlich dagegen vorgingen. ${ }^{5}$

* Raffaela Kunz ist Referentin am Max-Planck-Institut für ausländisches öffentliches Recht und Völkerrecht und Co-Chefredakteurin des Völkerrechtsblogs. Michael Riegner ist wissenschaftlicher Mitarbeiter an der Humboldt-Universität zu Berlin und Mitbegründer des Völkerrechtsblogs. Dana Schmalz ist New York/Feodor Lynen Fellow der Alexander von Humboldt-Stiftung an der Columbia Law School und Co-Chefredakeurin des Völkerrechtsblogs.

1 Klaus Graf, Vermitteln Blogs das Gefühl rastloser Masturbation? Eine Antwort auf Valentin Groebner, Redaktionsblog, 7. Februar 2013, http://redaktionsblog.hypotheses.org/951 (zuletzt besucht am 19. März 2019).

2 Siehe etwa die Studie von Carol Watson und James M. Donovan, Citation Advantage of Open Access Legal Scholarship, Law Library Journal 103 (2011), 553-573.

3 WISSENSCHAFTSRAT, Empfehlungen zu Forschungsinfrastrukturen in den Geistes- und Sozialwissenschaften (Berlin: Wissenschaftsrat 2011), 8.

4 WISSENSCHAFTSRAT, Perspektiven der Rechtswissenschaft in Deutschland. Situation, Analysen, Empfehlungen (Hamburg: Wissenschaftsrat 2012), $66 \mathrm{ff}$.

5 Siehe den offenen Brief vom 1. Februar 2016, einsehbar unter http://www.ordnungderwissenschaft.de /2016-2/07_Infoteil/14_loewisch_konstanzer-juristenfakultaet_verweigert_zweitveroeffentlichungspfli 
Eine Ausnahme bilden rechtswissenschaftliche Blogs, die als genuin digitale und frei zugängliche Medien regelmäßig als wichtige Akteure ${ }^{6}$ oder gar ,goldener Lichtstreifen "7 in der ansonsten Open-Access-skeptischen Rechtswissenschaft gesehen werden. Ursprünglich vor allem in den Vereinigten Staaten verbreitet sind Blogs - zumindest im Bereich des öffentlichen Rechts - inzwischen auch im deutschsprachigen Raum zu einem zentralen Ort der Wissenschaftskommunikation geworden und aus der Wissenschaftslandschaft nicht mehr wegzudenken. Neben dem Völkerrechtsblog (www.voelkerrechtsblog.org), der im Rahmen dieses Beitrages kurz vorgestellt werden soll, sind für das öffentliche Recht der Verfassungsblog (www.verfassungsblog.de), der dieses Jahr sein zehnjähriges Bestehen feiern kann, sowie JuWiss (www.juwiss.de), ein Nachwuchsforum, zu nennen.

Dass Blogs in der Völkerrechtswissenschaft inzwischen einen wichtigen Stellenwert einnehmen, ist insofern wenig erstaunlich, als der Forschungsgegenstand der Völkerrechtswissenschaft naturgemäß ein grenzüberschreitender ist und die internationale Sichtbarkeit von Forschenden eine größere Rolle spielt. Einer der Hauptbeweggründe für die Gründung von Völkerrechtsblog war es denn auch, die neuen Räume und Möglichkeiten, die das Internet eröffnet, für die Völkerrechtswissenschaft fruchtbar zu machen und einen grenzüberschreitenden Diskurs zu fördern, der dem Universalitätsanspruch des Völkerrechts entspricht.

Im Folgenden soll kurz die Entstehungsgeschichte des Völkerrechtsblogs rekapituliert werden (B), bevor in einem nächsten Schritt der Blog vorgestellt und erläutert wird, inwiefern sich dieses Publikationsformat von anderen abhebt (C). Der Beitrag schließt mit einigen Überlegungen zur Zukunftsvision des Völkerrechtsblogs (D).

\section{B. Entstehungsgeschichte}

Nachdem andere Projekte den Weg geebnet haben, wurde der Völkerrechtsblog 2014 im Rahmen des Arbeitskreises junger VölkerrechtswissenschaftlerInnen (AjV) gegründet. Der AjV ist ein informelles Netzwerk, das zum Ziel hat, VölkerrechtlerInnen der jüngeren Generation aus Deutschland, Österreich und der Schweiz zu vernetzen. ${ }^{8}$ Die Mitglieder des AjV verbindet, dass sie nach 1990 studiert haben und entsprechend einer Generation angehören, die den technischen Wandel unmittelbar miterlebt hat und sich den neuen Herausforderungen neugierig stellt.

cht_odw_2016.pdf (zuletzt besucht am 19. März 2019). Das Verwaltungsgericht Baden-Württemberg hat die Frage mit Entscheidung vom 26. September 2017 dem Bundesverfassungsgericht vorgelegt.

6 Siehe auch Siggener Thesen zum wissenschaftlichen Publizieren im digitalen Zeitalter (https://www.m erkur-zeitschrift.de/2016/10/24/siggenthesen/), \#Siggenthesen Nr. 5: „Webmedien wie Blogs, Wikis und andere soziale Medien sind zentral für einen offenen und freien Wissenschaftsdiskurs.“.

7 Hanjo Hammann, Open Access in der Rechtswissenschaft. Tagung an der Universität Bern am 27.5.2016, GRUR 2016, 1140-1142, 1141.

8 Siehe für mehr Informationen https://voelkerrechtsblog.org/community/ajv/internal/mitgliederliste/. 
Ziel war es also, die potenziell globale virtuelle Öffentlichkeit im Netz zu nutzen und einen transnationalen wissenschaftlichen Diskurs zu fördern. Denn wie bereits erwähnt ist in der Völkerrechtswissenschaft eine grenzüberschreitende Wissenschaftskommunikation eine Frage der diszipoinären Identität. Der Gegenstand der Völkerrechtswissenschaft ist definitionsgemäß nicht auf einzelne Rechtsordnungen beschränkt. Trotzdem wird ein wirklich transnationaler Wissensfluss und Diskurs auch in dieser Teildisziplin durch national ausgerichtete Publikationen und Bezahlschranken behindert, denn Open Access hat sich auch in der Völkerrechtswissenschaft nach wie vor nicht durchgesetzt - mit Folgen insbesondere für Forschende weniger wohlhabender Forschungseinrichtungen im Globalen Süden. Dies stellt nicht nur die Internationalität des internationalen Rechts bis heute in Frage, ${ }^{9}$ sondern ist auch ein Erkenntnishindernis in der Völkerrechtswissenschaft.

Auf diese Probleme versprach das Internet neue Antworten zu liefern. Bei der Gründung von Völkerrechtsblog fiel die Entscheidung ganz bewusst auf das Medium Blog, das mit seiner Niederschwelligkeit nicht nur ein breites Publikum zu erreichen verhieß. Über Wordpress war der Betrieb eines Blogs zudem mit wenig technischem Aufwand und Vorwissen und ausgesprochen kostengünstig möglich. So startete das Projekt Völkerrechtsblog quasi am Küchentisch als Initiative einer Gruppe befreundeter Doktorierender.

\section{Was ist der Völkerrechtsblog? Ein Überblick}

Das Profil des Völkerrechtsblogs ist vor diesem Hintergrund zu sehen. Wichtig ist zunächst, dass sich der Völkerrechtsblog als sog. Wissenschaftsblog versteht, d.h. als Blog, der aus der Rechtswissenschaft heraus betrieben wird und den Anspruch hat, sich an Diskussionen in der Fachgemeinschaft und am wissenschaftlichen Erkenntnisgewinn zu beteiligen. ${ }^{10}$ Im Unterschied zu anderen Blogs zielt der Völkerrechtsblog nicht primär auf Tagesaktualität ab, sondern sieht sich als „zweite Reflexionsschleife" mit theoretischem Anspruch, der zwischen Nachrichtenblogs und wissenschaftlicher Zeitschrift angesiedelt ist, auch aktuelle wissenschaftliche Debatten aufnimmt und einem größeren Publikum zugänglich macht („Slow Blogging “). ${ }^{11}$ Zur wissenschaftlichen Qualitätssicherung setzt die Redaktion auf ein schlankes Peer-Review-Verfahren durch einen wissenschaftlichen Beirat, der aus zumeist promovierten Mitgliedern des wissenschaftlichen Nachwuchses besteht. ${ }^{12}$

9 Vgl. Anthea Roberts, Is International Law International? (Oxford: Oxford University Press 2017).

10 Siehe für eine begriffliche Diskussion Hannah Birkenkötter, Blogs in der Wissenschaft vom Öffentlichen Recht. Ein Beitrag zur Erschließung neuer Formate, in Andreas Funke \& Konrad Lachmayer (Hrsg.), Formate der Rechtswissenschaft (Weilerswist: Velbrück Wissenschaft 2017), 117-139, $125 \mathrm{ff}$.

11 Siehe für einen kurzen Überblick Dana Schmalz und Michael Riegner, Völkerrecht 2.0 - es ist angerichtet, Völkerrechtsblog, 29. April 2014, doi: 10.17176/20170104-153908.

12 Für einen Überblick siehe https://voelkerrechtsblog.org/about/team-and-contact/. 
Dies stellt die Rückbindung an klassische Formen und etablierte Vertreterinnen der Völkerrechtswissenschaft sicher und macht deutlich, dass es nicht Ziel des Völkerrechtsblogs ist, mit den bestehenden Strukturen zu brechen. Vielmehr versuchen wir existierende Formate zu ergänzen und sogar Brücken zwischen klassischen und neueren Formaten zu schlagen.

Im Zentrum des Projekts steht die Idee des Dialoges und Austausches. Der Völkerrechtsblog versteht sich als Plattform, auf der Ideen ausprobiert und weiterentwickelt werden können mit dem Ziel, gemeinsam am Erkenntnisgewinn zu arbeiten. Entsprechend sind Beiträge auf dem Blog mit 1000-1500 Wörter kürzer als herkömmliche Formate und dürfen auch thesenhafter und weniger formell als klassische Zeitschriftenbeiträge sein. Beiträge auf dem Blog erscheinen zudem in deutsch, englisch oder französischer Sprache, um ein möglichst breites Publikum zu erreichen.

Das dialogische Element widerspiegelt sich in den verschiedenen Beitragskategorien auf dem Blog. „Markenzeichen“ des Völkerrechtsblogs ist es, dass Beiträge in der Regel „im Doppelpack“ erscheinen: In einem ersten Schritt erscheint ein Auftaktsbeitrag, auf den eine später erscheinende Replik Bezug nimmt. Auch im Rahmen der Zeitschriftenkooperation, einem Format, das der Völkerrechtsblog gänzlich neu entwickelt hat, steht der wissenschaftliche Austausch im Vordergrund: Im Rahmen von Partnerschaften mit renommierten Fachzeitschriften - bislang Verfassung und Recht in Übersee (VRÜ), Archiv des Völkerrechts, dem Leiden Journal of International Law (LJIL), der Swiss Review of International and European Law (SRIEL) und dem Nordic Journal of International Law ${ }^{13}$ - laden wir AutorInnen mit einschlägiger Expertise ein, aktuell in diesen Zeitschriften erschienene Beiträge auf dem Völkerrechtsblog zu diskutieren und diese dadurch einem breiteren Publikum zugänglich zu machen. Die in Bezug genommenen Zeitschriftenbeiträge werden von den Verlagen in der Regel online frei zugänglich gemacht, so dass der Blog positive Rückwirkungen im Sinne von Open Access in traditionellen Medien zeitigt. ${ }^{14}$ Diese Zusammenarbeit erhöht die Akzeptanz und Sichtbarkeit in der Fachgemeinschaft. Ein weiteres Format, das klassische mit neuen Publikationsformen verbindet, ist das des Buchsymposiums: Dabei diskutieren RezensentInnen mit dem Autor oder der Autorin eines Buches auf dem Blog direkt über das Werk. ${ }^{15}$ Ähnliche Brücken schlagen Konferenzsymposien. ${ }^{16}$

13 Siehe unter http://voelkerrechtsblog.org/about/partners-and-sponsors/.

14 Namentlich VRÜ und LJIL stellen auf dem Völkerrechtsblog diskutierte Artikel Open Access. Siehe für ein jüngeres Beispiel Iris Canor, Is a bird in the hand always worth two in the bush?, Völkerrechtsblog, 13. September 2017, doi: 10.17176/20170913-105951.

15 Vgl. z.B. http://voelkerrechtsblog.org/category/symposium/mollers-buchforum/.

16 Ein Beispiel hierfür ist das Symposium „Global South and Comparative Constitutional Law“ zur gleichnamigen Konferenz an der Humboldt Universität zu Berlin. Siehe https://voelkerrechtsblog.org /category/symposium/global-south-in-comparative-constitutional-law/. 
Neben der Publikation inhaltlicher Beiträge unterhält der Völkerrechtsblog ferner eine Service-Sektion, die monatlich über Stellenausschreibungen und Veranstaltungen im Bereich des Völkerrechts im deutschsprachigen Raum informiert. Diese Leistung wird von einem eigens zuständigen vierköpfigen Team erbracht. Seit 2016 kooperiert das Service-Team mit der Deutschen Gesellschaft für Internationales Recht (DGIR) und stellt dieser die Inhalte der Service-Sektion für einen Newsletter aufbereitet zur Verfügung.

In rechtlicher Hinsicht nutzt Völkerrechtsblog für die inhaltlichen Beiträge den auch von anderen rechtswissenschaftlichen Blogs verwendeten Standard-Lizenzvertrag Creative Commons Share Alike 4.0 (Namensnennung). Die Beiträge auf Völkerrechtsblog werden mit einem stabilen Digital Object Identifier (DOI) versehen und mithilfe des Open-Access-Fachrepositorium <intR $>2$ Dok an der Staatsbibliothek Berlin langzeitarchiviert und bibliothekarisch nachweisbar gemacht. Zudem bewerben wir sämtliche Inhalte auf Twitter und Facebook. Seit Sommer 2018 ist ein Redaktionsmitglied ausschließlich für die Social-Media-Präsenz zuständig, und der Eindruck ist, dass sich dies positiv auf die Sichtbarkeit der Beiträge und des Blogs insgesamt auswirkt. Seit wir Twitter aktiv betreuen, haben wir ca. 1500 Follower dazugewonnen (Stand Oktober 2019: 2700 Follower) und gemäß unseren internen Statistiken sind in diesem Zeitraum auch unsere Leserzahlen überdurchschnittlich gestiegen. Gerade Twitter dient aber nicht nur zur Verbreitung von Beiträgen; inzwischen findet auf dieser Plattform häufig auch inhaltlicher Austausch über einzelne Beiträge und Argumente statt. Es ist auch schon vorgekommen, dass ein Austausch via Twitter Autoren zu einer formellen Replik auf dem Völkerrechtsblog motiviert hat.

Fünf Jahre nach seiner Gründung ist der Völkerrechtsblog eine erfolgreiche Institution und Wissenschaftsinfrastruktur, die nicht nur international gelesen, sondern auch beforscht wird. ${ }^{17}$ Auf dem Blog wurden bis dato rund 600 Beiträge veröffentlicht; der Publikationsrhythmus ist konstant hoch (rund drei Beiträge pro Woche). ${ }^{18}$ Die Autorenschaft umfasst sowohl Mitglieder des wissenschaftlichen Nachwuchses als auch etablierte WissenschaftlerInnen aus allen Weltregionen. Die internen Statistiken zeigen, dass Beiträge durchschnittlich rund 250 Leser erreichen, wobei einzelne Beiträge Leserzahlen bis weit in den vierstelligen Bereich erzielen. ${ }^{19}$ Vom Erfolg des Blogs zeugt auch, dass die Redaktion in zunehmendem Umfang Initiativeinsendungen zur Publikation erreichen. Die Leserdaten belegen dabei ein-

17 Siehe aus jüngerer Zeit etwa Antoine Duval, 'Publish (Tweets and Blogs) or Perish? Legal Academia in Times of Social Media' (2018) 23(1) Tilburg Law Review pp. 91-108, DOI: https://doi.org/10.53 34/tilr.4.

18 Interne Wordpress-Statistik, Stand März 2019.

19 Der zurzeit mit rund 10‘300 Lesern meistgelesene Beitrag ist Valentin J. Schatz and Dmytro Koval, Ukraine v. Russia: Passage through Kerch Strait and the Sea of Azov (Part I), Völkerrechtsblog, 10. Januar 2018, doi: 10.17176/20180110-131019. 
drücklich, dass elektronische und frei zugängliche wissenschaftliche Publikationen tatsächlich quasi weltweit rezipiert werden: Die digital erfasste Leserschaft des Völkerrechtsblogs stammt aus über 180 Ländern, wobei neben den deutschsprachigen Ländern unter anderem die USA, die Türkei, Indien, Brasilien und Südkorea zu den Ländern zählen, in denen der Blog am häufigsten gelesen wird. ${ }^{20}$

\section{Zukunftsvisionen}

So erfreulich diese Entwicklung ist, so sehr drohte das Projekt Völkerrechtsblog jedoch zunehmend Opfer seines eigenen Erfolges zu werden. Aus dem kleinen Kreis von Doktorierenden, der am Anfang stand, wurde zwar eine rund 15-köpfige Redaktion, die inzwischen auch Postdocs einschließt. Zusätzlich kümmert sich ein vierköpfiges Team um die Service-Sektion. Trotzdem funktionierte der Blog nach wie vor vorwiegend auf ehrenamtlicher Basis. Finanzielle Unterstützung erhielt der Völkerrechtsblog lediglich auf kleiner Basis; so beteiligt sich die DGIR mit 80 Euro monatlich an den Kosten des Blogs. Zudem übernahm das Max-Planck-Institut für ausländisches öffentliches Recht und Völkerrecht (MPIL) aus Mitteln des an Armin von Bogdandy verliehenen Leibniz-Preises die Fahrtkosten der Redaktionsmitglieder zu den zwei Mal jährlich in Berlin stattfindenden Redaktionstreffen sowie die Kosten für Wordpress sowie einige kleinere Programmierleistungen. Dieses auf Freiwilligkeit und Ehrenamt basierende Modell erlaubte es jedoch das bestehende Potenzial nicht annähernd auszuschöpfen und der Völkerrechtsblog stieß personell und organisatorisch zunehmend an seine Grenzen.

Erfreulicherweise ist es gelungen, in Zusammenarbeit mit dem Max-Planck-Institut für ausländisches öffentliches Recht und Völkerrecht Mittel im Förderprogramm „Infrastruktur für elektronische Publikationen und digitale Wissenschaftskommunikation" der Deutschen Forschungsgemeinschaft (DFG) einzuwerben. ${ }^{21}$ Diese Förderung finanziert seit März 2019 u.a. eine Postdoc-Stelle, die das Projekt hauptamtlich koordiniert, und zwei studentische Hilfskräfte, die mit je fünf Wochenstunden am Projekt mitarbeiten.

Die dreijährige Förderung soll primär dazu genutzt werden, das Projekt Völkerrechtsblog weiter zu professionalisieren und zu profilieren. Noch stärker als bislang soll der Völkerrechtsblog inhaltliche Themen und Impulse setzten und die Diskussion in der Fachgemeinschaft mitprägen. Dazu gehört auch eine Modernisierung der Website und unserer Organisationsstruktur sowie die Entwicklung ei-

20 Worpdress-Statistik des Völkerrechtsblogs.

21 Wissenschaftsblogs als Infrastruktur für digitale Publikationen und Wissenschaftskommunikation: Ausbau des Modellprojekts „Völkerrechtsblog“, einsehbar unter http://gepris.dfg.de/gepris/projekt/4 07446657 (besucht am 18. März 2019). Siehe dazu auch Dana Schmalz, Michael Riegner and Raffaela Kunz, Funding Völkerrechtsblog. The German national research funding organization DFG will support Völkerrechtsblog in the coming years, Völkerrechtsblog, 5. Dezember 2017, doi: 10.17176/20181205-111550-0. 
nes längerfristigen und nachhaltigen Finanzierungsmodells. Die Finanzierung bleibt dabei die Gretchenfrage aller Blogs und von Open-Access-Formaten generell. Als Antwort kommen mehrere Modelle in Betracht. Dazu gehört zum einen eine noch engere Zusammenarbeit mit einer Forschungseinrichtung. Selbstredend bleibt die inhaltliche Unabhängigkeit unabdingbare Voraussetzung jeglicher institutionellen Zusammenarbeit. Ebenfalls denkbar ist eine verstärkte Einwerbung eigener Drittmittel durch Beteiligung an wissenschaftlichen Forschungsprojekten oder eine (Teil-)Kommerzialisierung, etwa der Stellenanzeigen und Servicesektion. All diese Modelle haben jeweils Vor- und Nachteile, so dass im Idealfall eine Kombination anzustreben ist, die inhaltliche Unabhängigkeit, wissenschaftliche Qualität und nachhaltige Finanzierung gleichermaßen sichert.

Ein wichtiges Anliegen ist und bleibt uns die weitere Öffnung und Erweiterung des Diskussionsraums insbesondere auch über Europa hinaus und bis in den Globalen Süden. Insbesondere geht es darum, WissenschaftlerInnen aus dem Globalen Süden nicht nur als Rezipienten, sondern auch als Produzenten zu gewinnen. Auch das erfolgreiche Format der Zeitschriftenkooperation soll durch Kooperationen mit Zeitschriften aus dem Globalen Süden erweitert werden. Und natürlich möchte sich der Völkerrechtsblog auch weiterhin an der Debatte um Open Access in der Rechtswissenschaft beteiligen mit dem Ziel, diese voranzutreiben. So wird der Völkerrechtsblog eine internationale Konferenz ausrichten, die speziell die Perspektive der Völkerrechtswissenschaft in der Open-Access-Debatte beleuchten soll. Auch wenn wir uns dabei in erster Linie der Sache verpflichtet fühlen, geschieht all dies letztendlich nicht ganz uneigennützig, sind wir doch davon überzeugt, dass die Sichtbarkeit auch der deutschen Völkerrechtswissenschaft längerfristig von Open Access abhängt. 